\title{
Las relaciones hispano-británicas tras la paz de Versalles (1783)
}

\author{
ana Clara Guerrero
}

La firma en septiembre de 1783 del tratado de Versalles puso fin al conflicto entre Gran Bretaña y sus colonias en América del Norte, que, iniciado en las postrimerías de la Paz de París, 1763, se convirtió en enfrentamiento armado en 1776 y en guerra internacional poco después. Si París había simbolizado el hundimiento del primer imperio colonial francés y el triunfo del expansionismo británico, el reconocimiento en Versalles, veinte años después, de la independencia de las trece colonias supuso un duro golpe para el prestigio británico. Privada de parte de su Imperio en América, sin aliados y con sus finanzas en un estado caótico, Gran Bretaña se enfrentaba a un período difícil. A pesar de que la victoria inglesa en la batalla de los Santos, poco antes del fin de la guerra, era una clara prueba de que, aunque derrotada, Gran Bretaña conservaba prácticamente intacto su poderío naval, en algunos círculos se extendió la idea de que esta primera manifestación de debilidad de los Hannover era quizás el inicio del esperado declive del imperio británico.

El primer ministro encargado de dirigir el país en estos años difíciles era el joven William Pitt. Hijo del político que sacó a Gran Bretaña de la crisis moral en que se vio sumida tras el largo gobierno de Walpole, y que muchos han considerado fundador del primer imperio británico, el joven Pitt llegaba al poder con tan sólo veinticuatro años y entre la desconfianza de gran parte de los políticos de la época, aunque en aquellas fechas ya era miembro del Parlamento y había sido Canciller del Tesoro bajo Shelburne. Pitt tenía que enfrentarse a los problemas que suponía un país aislado diplomáticamente, desmoralizado y con una deuda nacional que se había duplicado durante la guerra americana. La actividad que desplegó para solucionarlos le granjeó en muy poco tiempo la admiración 
y el apoyo de muchos de aquellos que en un principio le habían observado con reticencia. La habilidad con que supo dirigir sus asuntos y los del país le permitió permanecer en el puesto de Primer Ministro prácticamente toda su vida, condenando al ostracismo al otro gran político del período, su rival Charles James Fox.

\section{POLITICA EXTERIOR Y COMERCIO}

Desde el mismo instante de su llegada al poder Pitt dejó patente que su principal preocupación sería la recuperación financiera y económica del país. Sin embargo, esto no significaba el abandono en un segundo plano de las relaciones internacionales, que se centrarían en la búsqueda de nuevos aliados y el fortalecimiento de los lazos con el continente. El Secretario de Estado designado para Asuntos Exteriores, Camarthen, pasó en seguida a ser conocido en las cancillerías europeas como «la marioneta de Pitt" ${ }^{1}$, pues la sombra del Primer Ministro estaba detrás de cualquier iniciativa emprendida por el Foreign Office. La reforma económica que tanto preocupaba a Pitt estaba en algunos aspectos estrechamente unida a la política internacional. Gran Bretaña necesitaba asegurarse la llegada de materias primas para su floreciente industria y, sobre todo, mercados para sus productos manufacturados. En sus negociaciones eni búsqueda de aliados, Pitt y Camarthen no olvidaban los intereses económicos nacionales. Las alusiones a la necesidad de «buenas relaciones entre las naciones», referidas fundamentalmente a las «buenas relaciones comerciales" serán constantes en estos años de lucha pacífica por salir del aislamiento. Prueba de esta preocupación que vinculaba intereses diplomáticos y económicos fue la proliferación de negociaciones destinadas a la firma de acuerdos comerciales. En los años que siguieron a la paz de Versalles se iniciaron conversaciones con Portugal, Rusia, Francia, España, Suecia, Turquía, los Países Bajos Austriacos y las Dos Sicilias $^{2}$.

La admiración de William Pitt por las ideas de Adam Smith, de quien el primer ministro se consideraba discípulo, hizo creer en muchos secto-

\footnotetext{
1 P. Langford, The Eighteenth century, 1688-1815. Londres 1976, pág. 189.

${ }^{2}$ La evolución de estas negociaciones ha sido estudiada por J. EHRMAN. The British Government and commercial negotiations with Europe. 1783-1793. Cambridge 1962.
} 
res que se iniciaba un nuevo período en la historia de las relaciones comerciales que se caracterizaría por el predominio de las ideas librecambistas frente a las mercantilistas. La reciprocidad y la justa competencia terminarian con los monopolios y las restricciones que habían caracterizado épocas anteriores. Determinadas medidas económicas adoptadas durante el gobierno Pitt estaban directamente inspiradas en las ideas contenidas en La riqueza de las Naciones, por ejemplo su decisión de no prohibir la importación de grano extranjero durante los años de guerra. Esta apertura gubernamental hacia nuevas ideas abrió interesantes expectativas en algunos de los países que negociaban con Gran Bretaña, entre ellos España, deseosa de una redefinición de sus relaciones económicas que implicase una mayor reciprocidad. 'El tiempo se encargaría de decepcionarles. En el fondo de estas buenas intenciones, detrás de todas las manifestaciones llamando a la "armonía comercial», subyacía una idea fundamental: Pitt quería devolver a Gran Bretaña su poderío, su posición de potencia imperial. España fue uno de los primeros en darse cuenta de que la supuesta debilidad británica era sólo un espejismo y de que las manifestaciones librecambistas eran más una defensa de intereses que una proclamación de principios.

\section{LA MONARQUÍA «MÁS MISTERIOSA, CELOSA Y TAIMADA DE EUROPA ${ }^{3}$}

En 1783 se presentaba al Secretario de Estado un informe sin firma, de casi treinta páginas, en el que se analizaban aquellos elementos que deberian tenerse en cuenta de cara al establecimiento de relaciones con los Borbones españoles tras la guerra de independencia de las colonias de América del Norte ${ }^{4}$. El autor parte de una serie de premisas funda-

${ }^{3}$ PRO: FO 72/1. Some memoranda for treating with Spain. El autor de este largo informe afirma que se conoce mucho menos sobre España que sobre otros países y lo atribuye al menor número de viajeros que la han recorrido y al carácter de su Corte, "la más misteriosa, celosa y taimada de Europa".

4 PRO: FO 72/1. Some memoranda for treating with Spain. Aunque este extenso informe carece de firma, una serie de elementos permiten atribuirlo a ALEXANDER JARDINE. Aunque es conocido sobre todo por la interesante amistad que en los últimos años de su vida (1793-99), cuando fue cónsul británico en España, mantuvo con Jovellanos y de la que ha quedado constancia en los Diarios del famoso ilustrado, los contactos de Jardine con este país se remontan a un período anterior. Militar de carrera, fue enviado a Gibraltar durante la guerra de los Siete Años para participar en su defensa, permaneciendo en el peñón una 
mentales que no son más que una exposición de los principios que, bajo la inspiración de Pitt, regían la política europea del Foreign Office. En primer lugar, la «defensa del papel de potencia de primera fila» que sigue correspondiendo a Gran Bretaña. Para ello hay que luchar contra la opinión extendida en España, y en otros países, de que tras la pérdida de parte de sus colonias Gran Bretaña se encuentra "abatida y en declive». Todos los representantes británicos insistirán en que su país continúa siendo "la primera potencia marítima", pues de su capacidad para dejar clara esta situación de «preeminencia» dependerá su «peso e importancia entre las naciones". En ningún caso podrá Gran Bretaña acceder a limitar su presencia en ningún mar; sería el primer paso hacia la decadencia. En segundo lugar y, una vez se haya dejado clara la fuerza británica, habrá que intentar "acabar con el aislamiento" en que se vio sumido el país como consecuencia de la guerra. "Como nación marítima y comercial no podemos vivir sin aliados", afirma el autor del informe. Los intereses británicos en Europa no han cambiado, incluso ahora - sin las colonias-Gran Bretaña se ve más necesitada de aliados y más forzada a tomar parte en los acontecimientos europeos. Pero no se trata de una búsqueda de alianzas a cualquier precio. Pitt pretende una política exterior rentable, que no se convierta en una fuente de gastos incalculable, como ocurrió en otras épocas a algunos imperios. En este especial contexto, vinculando intereses políticos y económicos, se analizarán las posibles relaciones con España.

En caso de guerra y «con el propósito de comerciar», fin primordial para una «potencia marítima y comercial», los británicos deben «mirar

vez firmada la Paz de París. Desde este momento su vida quedó indisolublemente unida a la península. Dada su condición militar y experto en asuntos españoles, desempeñó varias misiones en la península, actuando como espía en el periodo inmediatamente anterior al estallido de la guerra de las colonias de América del Norte. En el grueso volumen formado por documentos correspondientes a 1781-83 conservado en el Public Record Office, encontramos junto al informe antes citado algunas cartas de Jardine, fechadas en los últimos meses de 1782 y primeros del 83 . En ellas el militar recuerda los servicios prestados a su Corona y señala que se le pidió «ordenar y hacer un resumen de mis últimas observaciones militares e informes, fruto de mi último viaje y residencia en España, algunos de los cuales ya deben estar en su oficina. El resto del material obra en mi poder, pues no se me ha solicitado" (PRO: FO 72/1). Si esta afirmación de Jardine podría hacernos pensar en una posible relación entre sus cartas y el informe que las sigue, el paralelismo existente entre algunas de las opiniones en él vertidas y las expresadas en su obra Letters from Barbarie, France, Spain, Portugal, etc. aparecida en Londres en 1788, proporciona más argumentos a favor de la atribución a Jardine del informe anónimo. En él se trasluce ya el liberalismo y el sentimiento antiborbónico que caracterizarán sus Letters y cuando el autor analiza la importancia de Gibraltar para los británicos lo hace con argumentos que reproducirá años después en su libro (Ver PRO: FO 72/1 y op. cit. Vol. II. Págs. 331-334). 
hacia el sur tanto como hacia el norte». Dada la inevitable enemistad con los Borbones, España aparece como el «enemigo natural» de Gran Bretaña en la zona y Portugal como el «amigo natural». Pese al interés británico por debilitar la alianza borbónica, las probabilidades de que haya cambios en las relaciones con esta región europea se presentan remotas. Sin embargo, y aun en el caso de futuras guerras, este pacto familiar no supondría un gran riesgo a juicio del autor del informe, pues «(...) es mejor tener a España contra nosotros, que a nuestro favor, mejor que sea un enemigo débil que un amigo débil, mejor que sea una carga para nuestros enemigos que para nosotros, mejor que sea el miembro débil y vulnerable de una confederación en contra nuestra, que encontrarla en cualquier otra posición”.

Pero, aunque en el terreno político la batalla frente a la influencia francesa en España deba ser dada por perdida, Gran Bretaña tiene que luchar por conseguir mantener y acrecentar, si fuera posible, su preeminencia comercial en el sur de Europa. Sin las colonias americanas, «nuestra atención y nuestros capitales pueden y deben volverse hacia el comercio europeo y especialmente el del Mediterráneo". Ese mar, "en una situación y a una distancia tan adecuada, en el que 600100 millones de personas se comunican por sus necesidades y comercio, debe ser considerado de primera importancia para nosotros". Para asegurar la presencia británica en la zona el autor del informe recomienda mantener la amistad con Portugal, un acercamiento a los estados de la Berbería, con los que otras naciones europeas se niegan a mantener relaciones por considerarlos piratas, $y$, sobre todo, el control de algún puerto en el Mediterráneo. De acuerdo con estas premisas, defender el dominio británico sobre el peñón de Gibraltar, llave de entrada en dicho mar, sólida base para asegurar el control en la zona mediterránea se convertirá, para el autor de este informe, en el objetivo fundamental en cualquier negociación que se emprenda con España.

Las presiones españolas para recobrar Gibraltar habían arreciado como consecuencia de la derrota británica en la guerra contra sus colonias, hasta el punto de provocar divisiones en la Corte de San Jaime. Para algunos sectores, entre los que se encontraba el rey Jorge III, el peñón era sólo una causa de conflictos y estaban dispuestos a devolverlo si con ello se conseguía alejar a España de la alianza con Francia. Otros miembros del Gabinete y expertos vinculados al Foreign Office, entre los que se encuentra el autor de este informe, se negaban tajantemente a ceder esta plaza que la opinión popular había convertido en un símbolo del poderío británico. Temeroso de que el sector partidario de la devolu- 
ción de Gibraltar se hiciese con el control de la situación, el redactor de este "Memorandum» se lanza a un furibundo ataque para demostrar la invalidez de las posibles ventajas que tal cesión podría acarrear. En primer lugar, se niega a aceptar que la devolución de Gibraltar tenga los efectos que algunos han supuesto, pues «ni romperá el pacto de familia, ni cambiará en nada los fundamentos de la actual dependencia y relación, ni la presente situación establecida desde hace largo tiempo". "Saben muy poco de España y del mundo político, aquellos quienes crean que tendríamos más influencia sobre esta nación o sobre su corte sin Gibraltar en nuestras manos que con él.» En segundo lugar, preocupado por la influencia que las presiones de los comerciantes con intereses en España podrían ejercer sobre la actitud del Gobierno, llevándoles a una cesión del peñón a cambio de ventajas económicas, dedica un buen número de páginas a intentar demostrar la escasa importancia que España reviste para Gran Bretaña, sobre todo en el campo de las relaciones comerciales.

Para el autor del informe no hay atisbos de que este país pueda «recuperar su grandeza, peso o importancia, ni aumentar en riquezas o fuerza, aunque intenten engañarse a sí mismos y a algunos otros por medio de regulaciones de comercio en parte adecuadas y en parte inefectivas, (...); tenemos poco que esperar o temer de su comercio o su poder, excepto como aliado fiel pero débil de Francia". Partiendo de esta afirmación de decadencia, que responde a la imagen generalizada que de España se tenía en la Inglaterra del siglo XVIII, realiza, de forma muy sintética, un análisis de los principales problemas que aquejan a dicho país, coincidiendo en algunos puntos con los ilustrados españoles. La agricultura, "absolutamente necesaria para la prosperidad nacional» tiene serios problemas para salir del atraso en que se encuentra por la inmovilidad de la tierra, la falta de capitales para invertir en mejoras y la ausencia de mercado para sus productos. La inexistencia de caminos apropiados pese a los esfuerzos realizados en los últimos años y a la gran cantidad de dinero en ellos empleados, constituye un obstáculo insuperable para el desarrollo de cualquier tipo de comercio. Esta grave dificultad, unida a un sistema fiscal opresivo y abusivo, con impuestos como la alcabala — capaz por sí sola de hundir y arruinar a cualquier nación y su comercio-, impiden el desarrollo de las manufacturas. Si a ello unimos las ideas erróneas que sobre la industria advierte en el país y la actitud de sus dirigentes, concentrados en desarrollar con capital real manufacturas demasiado grandes y, olvidando que sólo «la libertad y la seguridad hacen que la gente sea industriosa y previsora de cara al futuro y atraen capitales hacia las manufacturas» resulta altamente impro- 
bable que sus proyectos se vean acompañados por el éxito y lleguen a constituir una preocupación para Gran Bretaña. Pero este análisis de la situación económica española no muy alejado de la realidad y que, en mayor o menor medida según periodos, fue compartido por ilustrados españoles, aparece unido en el informe a una serie de afirmaciones sobre la escasa importancia de España como proveedor y consumidor de productos británicos que resultan ser la parte más débil de su argumentación. Su justificado temor a la capacidad de presión de los comerciantes y manufactureros británicos sobre el gobierno y las consecuencias que la defensa de intereses económicos inmediatos podía tener en una política más global y a largo plazo, le lleva a afirmar, frente a toda lógica y en contra de la realidad, que el papel desempeñado por España en el sistema comercial británico puede ser fácilmente ocupado por Portugal y que las dificultades que pudieran surgir como consecuencia de la carencia de algunos productos fundamentales, por ejemplo la lana, encontrarían fácil solución en un país con una capacidad agraria como la británica. Los únicos perjudicados en el caso de una ruptura de relaciones comerciales serían los propios españoles, que se verían privados de un buen cliente.

Este informe, redactado tras la paz de Versalles, proporciona la visión de un militar que recomienda al gobierno desconfiar de las opiniones de los comerciantes y hombres de negocios, quienes "siempre hablan teniendo sólo en cuenta su propio interés» y poniendo sus «deseos personales por encima del interés nacional». Se presenta a la España borbónica como el «enemigo natural» en una zona -el sur de Europa-por la que Gran Bretaña siente un gran interés. Pero, si bien interesa romper el aislamiento británico tras la guerra de las colonias americanas, no hay que olvidar la debilidad española y la búsqueda de unas relaciones más fiuidas, tanto en lo político como en lo económico, no debería hacerse a cualquier precio, especialmente si la moneda de pago resulta ser Gibraltar. La posesión de esta plaza, unida a la amistad de Portugal, los estados de la Berberia y otros países mediterráneos es, para el autor de este informe, fundamental para «recobrar nuestro peso y gloria nacional, algo que no se puede calcular en libras, chelines y peniques». 


\section{LA «MINA DE PLATA DE INGLATERRA» 5}

Sin embargo, las relaciones comerciales hispano-británicas tenían un peso muy superior al que este informe pretendía concederles. Desde la firma del Tratado de Madrid de 1667 España se habia convertido en un país fundamental para Gran Bretaña. Era el país europeo comprador de aquellos productos que los británicos deseaban vender $\mathrm{y}$, al mismo tiempo, era el proveedor de otros no menos importantes, sin olvidar el ansiado metal con que España cubría el déficit que esta relación desigual producía. El reconocimiento a los británicos en dicho tratado de todos los beneficios que hubiesen disfrutado anteriormente y la inclusión de algunos nuevos confería, sin duda, a Gran Bretaña el trato de «nación más favorecida" en sus relaciones con España. Comerciantes, políticos, diplomáticos, militares eran plenamente conscientes de esta ventajosa situación. Por ello, la sola mención del nombre de España en determinados círculos provocaba una inmediata asociación de ideas, era sinónimo de "negocio". La desaparición de los Habsburgo y la llegada al trono español de los Borbones no tuvo graves repercusiones, gracias a la hábil negociación llevada a cabo en Utrecht, pero sí acentuó en cambio la preocupación británica por mantener unas relaciones económicas fluidas con un país que quedaba vinculado al rival principal de Gran Bretaña, tanto en el escenario europeo como en el colonial. La especial alianza que se estableció entre las dos ramas de la Casa de Borbón hacían temer a Gran Bretaña, y con razón, la pérdida de la privilegiada situación comercial de que disfrutaba en beneficio de Francia, su principal competidor por el control del mercado español.

A lo largo del siglo ilustrado se sucedieron los enfrentamientos y las negociaciones de paz entre España y Gran Bretaña, yendo siempre los tratados acompañados de acuerdos comerciales en los que se renegociaban las relaciones económicas entre ambas naciones. La resolución a favor de la Gran Bretaña de los principales conflictos permitió a este país mantener su supremacía en los mercados españoles, pese a las trabas y cortapisas que el Gobierno de Madrid, por propio interés o respondiendo a presiones francesas, articulaba buscando frenar esta omnipresencia británica. La defensa de esta situación, en dura lucha diplomática con los franceses, fue un objetivo primordial de la política inglesa hacia España.

${ }^{5}$ Así calificaba a España el autor de un panfleto anónimo aparecidó en Londres en 1711 y titulado A proposal for humbling Spain written by a person of distinction. 
Los embajadores y enviados especiales, los cónsules destacados en las principales ciudades portuarias de la península dedicaban gran parte de su tiempo y esfuerzos a litigar con las autoridades españolas sobre supuestas o reales infracciones de los tratados vigentes: aplicación de tarifas inadecuadas, registros de barcos o confiscaciones de artículos juzgados improcedentes, repentinas prohibiciones de importación afectando a productos considerados esenciales por los comerciantes británicos, ..., páginas y páginas repletas de quejas que se amontonaban en los escritorios de los funcionarios españoles y que resultaban ser muy parecidas a las que recibían sus colegas británicos firmadas por españoles.

La política de desarrollo de las manufacturas peninsulares que caracterizó a los Gobiernos de los Borbones, comenzó a dar sus frutos a mediados de siglo, añadiendo un elemento más de preocupación para los británicos. Beawes, cónsul en Cádiz, Sevilla y Sanlúcar, afirmaba en 1754: «(...) lo que más ha perjudicado a nuestro comercio ha sido el establecimiento de multitud de fábricas para manufacturar bienes que los españoles antes obtenían fuera, principalmente de nosotros» ${ }^{6}$. Cada vez fueron más frecuentes los informes solicitados desde la Secretaría de Estado sobre la marcha de las principales manufacturas españolas o sobre las nuevas experiencias emprendidas directamente por el Estado o por particulares que se beneficiaban de la nueva política de fomento industrial. Sin embargo, los británicos pronto comprendieron que debían temer más a la actitud proteccionista de los gobiernos ilustrados peninsulares, que a las propias manufacturas españolas, incapaces de competir libremente con las procedentes de las Islas Británicas. En las fechas en que se firmó la Paz de Versalles España era aún el tercer cliente de los británicos en Europa, por delante de Portugal, el aliado británico por excelencia en el sur del continente ${ }^{7}$. A la hora de emprender negociaciones con España el Gobierno de William Pitt no podía olvidar esta realidad económica. Por otro lado los comerciantes se encargarían de recordárselo.

${ }^{6}$ W. BeAwES, Lex Mercatoria Rediviva or the Merchants directory. Dublin 1754, págs. 587-588.

${ }^{7}$ Para reconstruir la evolución de las cifras del comercio hispano-británico a lo largo del siglo XVIII son de especial interés los trabajos de L. PRADOs DE LA Escosura. Pueden verse, por ejemplo, los cuadros incluidos en su artículo «El comercio hispano-británico en los siglos XVIII y XIX. I. Reconstrucción" en Revista de Historia Económica. Año II. N. 2. (Primavera-Verano 1984). Págs. 113-162. 
En enero de 1783, los comerciantes británicos que negociaban con España enviaron un Memorial al Secretario de Estado ${ }^{8}$. En él, y ante la inminencia de negociaciones con España «humildemente" informaban a las autoridades de las innovaciones que en las regulaciones comerciales se habian ido introduciendo contrarias a los tratados vigentes y que eran "sumamente perjudiciales a los intereses comerciales de este Reino». Sus quejas principales se referían a las prohibiciones de importación de determinados artículos textiles, los aumentos de determinados impuestos, los problemas para establecer nuevos cónsules y los riesgos que correría un inglés protestante que pretendiese vivir y trabajar en una ciudad en la que no pudiese contar con el apoyo de un representante de Su Majestad. Su Memorial terminaba con la solicitud de que al negociar con España se obtuviera la seguridad de que todos los cambios introducidos contrarios a sus intereses serian suprimidos, sobre todo teniendo en cuenta que «en un momento de crisis y habiendo perdido el comercio británico sus exclusivas ventajas en los mercados americanos, el gobierno de Su Majestad debe, en función del interés nacional, tener muy en cuenta el aumento y la estabilidad del comercio exterior».

La política diseñada en el Foreign Office para sus relaciones con España intentaría conjugar estos dos enfoques, que no eran totalmente opuestos. Así, reconoció la importancia de las relaciones comerciales con la península y sus colonias, sin dejar por ello de tener muy en cuenta el papel fundamental que la posesión de Gibraltar había adquirido en la política imperial británica.

\section{LISTON Y FLORIDABLANCA}

Tras la firma de la Paz de Versalles, el Gabinete británico decidió nombrar nuevo embajador ante la Corte española. Pero la llegada a España del representante supremo de la Corte de San Jaime se iría retrasando, lo que motivó que, en mayo de 1783, Robert Liston fuese nombrado Ministro plenipotenciario temporalmente, aunque continuó en su puesto durante más de cinco años, hasta agosto de 1788 cuando recibió la notificación de que había sido designado «enviado extraordinario» ante 1783.

${ }^{8}$ PRO: FO 72/1. Memorial of the British Merchants trading with Spain. 23 de enero de 
la Corte de Estocolmo ${ }^{9}$. Las instrucciones recibidas por Liston, así como las enviadas a candidatos a embajador que nunca llegaron a la Península, permiten hacerse una clara idea de la política española del Foreign Office. En ellas se insiste en tres objetivos fundamentales: vigilar para que nada afecte al comercio inglés en la península, preocuparse por la seguridad de la fortaleza de Gibraltar y no dejar pasar ninguna oportunidad de debilitar la alianza hispano-francesa, entablando estrechas relaciones con aquellos ministros que se muestren anglófilos. Todo ello sin olvidar enviar frecuentes informes dando cuenta de la situación española y de los posibles preparativos emprendidos por la Corte que pudiesen afectar a los británicos 0 sus aliados ${ }^{10}$. El cumplimiento de su misión durante cinco largos años llevó a Liston a establecer un estrecho contacto con el conde de Floridablanca.

Aunque ha quedado claramente demostrado el importante papel desempeñado por el rey Carlos III en la firma y defensa de los Pactos de Familia y su participación en el diseño de la política exterior española durante los primeros años de su reinado, los resultados de la Paz de París y la actitud de los franceses durante la crisis de las Malvinas fueron enfriando su interés por temas tan controvertidos, aun manteniéndose fiel a la alianza familiar. Cada vez más fueron sus Ministros los encargados de las relaciones exteriores de la nación, gozando de especial confianza - como en tantos otros temas - el conde de Floridablanca. Sin llegar nunca a la ruptura de la tradicional alianza con los Borbones franceses, la política exterior de Floridablanca estuvo caracterizada por los intentos de marcar distancias con el poderoso aliado, saliendo en lo posible de una dependencia que, en opinión de Moñino, había llegado a parecerse a la esclavitud. En septiembre de 1784 Robert Liston afirmaba en una carta cifrada al Secretario de Estado Camarthen: «Si se puede tener alguna esperanza de cambio, debe fundarse en el aumento de poder del conde Floridablanca y en su deseo de hacer a su país independiente de la alianza francesa. La influencia de este ministro sobre Su Majestad Católica ha llegado a ser tan grande que empieza a pensarse que posee autoridad despótica en su Departamento, y este Departamento tiene una especie de control sobre todos los demás" ${ }^{11}$.

9 PRO: FO 72/13. Whitehall. 22 de agosto de 1788.

${ }_{10}$ PRO: FO 72/1. Instructions to Robert Liston, esquire. Mayo 1783. Recommendations to Lord Chesterfield. Marzo 1784.

11 PRO: FO 72/3. De Liston a Camarthen. San lldefonso 16 de septiembre de 1784. 
Es difícil tomar al pie de la letra las manifestaciones de anglofilia con que Floridablanca alagaba a Liston en casi todas sus entrevistas, sin embargo, es evidente que la tarea de este último se vio muy facilitada por la tendencia del Ministro español a salir de la tutela francesa, estableciendo por su cuenta una amplia red de relaciones diplomáticas que alejasen la amenaza de conflictos bélicos. La esperanza de poder mantener fácilmente a España en una situación de neutralidad permitía a Liston dedicar sus esfuerzos a mejorar la situación del comercio británico en la península. En la Paz de Versalles se había incluido una claúsula por la que se dejaba abierta la puerta a una posible negociación sobre temas comerciales entre España y Gran Bretaña ${ }^{12}$. Fue una concesión a los deseos españoles de aprovechar cualquier oportunidad para reducir los privilegios comerciales británicos, estableciendo unas nuevas relaciones basadas en la "reciprocidad" de derechos. Sin embargo, Gran Bretaña demostró su fortaleza pese a la reciente derrota, negociando con firmeza en busca de «(...) fáciles y ventajosos acuerdos», un «aumento considerable en la exportación de nuestras manufacturas» y «recuperar nuestro floreciente comercio" ${ }^{13}$. La tendencia de Pitt y su gobierno a vincular intereses diplomáticos y económicos se puso claramente de manifiesto en la actuación de sus representantes en la península.

Mientras el Foreign Office dedicaba gran parte de su atención a los problemas centroeuropeos, las relaciones entre Liston y Floridablanca estuvieron marcadas por los intentos de lograr un borrador de acuerdo comercial aceptable por ambas partes ${ }^{14}$. El empeño resultó ser más difícil de lo que en un principio se podía pensar, a pesar del optimismo que en los primeros meses traslucían los informes de Liston a Camarthen. Chocaban intereses dificilmente conciliables. La parte española mostraba una lógica desconfianza hacia una llegada masiva y fácil de productos ingleses, durísimos competidores para las incipientes manufacturas que, con grandes

${ }^{12}$ El último tratado comercial entre ambas naciones había sido firmado en 1750 , siendo embajador británico en la península el famoso Benjamín Keene. En él se habían renovado las concesiones hechas a Gran Bretaña en el tratado de 1667, el más ventajoso que habían disfrutado. Acuerdos posteriores se habian limitado a confirmar textos anteriores.

13 PRO: FO 72/3. De Munro (cónsul general británico) a Camarthen. Madrid, 13 de diciembre de 1784 .

${ }^{14}$ Dos comisionados se encargaron de los contactos más directos: del Campo por parte española, y Woodford por la británica. Un análisis detallado de las negociaciones, con referencia a los principales puntos de litigio: impuestos, prohibición de importación de determinados productos británicos, acceso de los barcos españoles a los puertos ingleses, ... y a las posturas adoptadas por cada una de las partes puede verse en J. EHRMAN, Op. cit. 
dificultades y fuerte apoyo estatal, se estaban desarrollando en la península. Esta situación de inferioridad en el campo de las manufacturas y la necesidad de asegurarles un mercado era un caldo de cultivo espléndido para el desarrollo de posturas proteccionistas, difícilmente aceptables para unos británicos que se presentaban en este momento como los máximos defensores de las ideas de Adam Smith en el campo de las relaciones comerciales. Por otra parte, la pretensión española de recibir Gibraltar a cambio de concesiones comerciales - ya prevista por el Foreign Office- no encontrará eco en los negociadores británicos ${ }^{15}$.

La afirmación de que las relaciones comerciales entre países debían regirse por una libre y justa competencia será repetida hasta la saciedad por los negociadores británicos, sin conseguir aplacar los recelos hispanos. Para el representante británico la causa principal de las dificultades para llegar a un acuerdo estaba en la existencia en la península de numerosos «proyectistas», dedicados a sugerir los medios más apropiados para el desarrollo de las manufacturas, recomendando en su mayoría la prohibición de la salida de materias primas y la adopción de medidas proteccionistas. De entre las obras que circulaban en la España de la época destaca la de Bernardo Ward, pues, "Se me ha asegurado de buena fuente que la mayor parte de las ideas del conde de Floridablanca en materia de comercio y manufacturas de su país han sido tomadas de un libro, publicado hace algunos años, titulado Proyecto Económico" ${ }^{16}$. Preocupado por esta influencia y las repercusiones que podía tener en las negociaciones sobre el nuevo tratado, Liston envió a su superior un breve apunte biográfico de Ward, sin olvidar mencionar su origen irlandés, así como un resumen de sus principales ideas sobre el comercio y las manufacturas. Dejando de lado la afirmación del irlandés de que «La libertad es el alma del comercio y todo género de estanco su mayor contrario" ${ }^{17}$, Liston se centra en sus propuestas más mercantilistas, uniéndose al coro de voces que criticaban el atraso español en lo referente a la difusión de las nuevas doctrinas económicas.

En realidad, la decadencia que había experimentado la península, el atraso con que estaban intentando revitalizar sus manufacturas y su comercio, llevaban a un comportamiento ecléctico por parte de los gobier-

${ }^{15}$ Ver, por ejemplo, el informe que envia Liston sobre su respuesta a la propuesta de Floridablanca sobre Gibraltar en PRO: FO 72/4. De Liston a Camarthen. Madrid, 7 de enero de 1785.

16 PRO: FO 72/10. N. 29. De Liston a Camarthen. Aranjuez, 30 de junio de 1787.

17 B. Ward: Proyecto Económico. Madrid 1982 (1. a ed. 1779). Pág. 173. 
nos ilustrados. Junto con la constatación y el reconocimiento por parte de autores diversos de que medidas liberalizadoras en determinados aspectos de su comercio estaban dando a los ingleses la supremacía en este terreno, en la práctica se veía la necesidad de recurrir a una combinación de normas liberalizadoras, como la ley de libertad de comercio de granos, determinadas reformas aduaneras y fiscales, o las leyes en contra del sistema monopolístico de relaciones con América hasta estos años vigente - ley de 1778-, y de medidas proteccionistas de las aduanas exteriores para apoyar el incipiente desarrollo interno.

Por otra parte, pese a las referencias que desde altas instancias gubernamentales británicas se hacian a la obra y a las ideas de Adam Smith, la interpretación que muchos comerciantes y políticos de dicha nación hacían de la nueva ideología era un tanto personal. En amplios sectores «libertad de comercio" se traducía por «libertad para comerciar». En unas Observaciones sobre el comercio británico hacia España ${ }^{18}$ recibidas en 1785 en la Secretaria de Estado, junto a la solicitud de que en el nuevo tratado "se asegure la libertad de comercio", se defendía el mantenimiento de los antiguos privilegios «por ser absolutamente necesarios para el libre ejercicio del comercio". Las trabas y limitaciones puestas en estos mismos años al establecimiento de una fluidas relaciones comerciales con sus ex-colonias de América del Norte eran una buena prueba de que no había inconveniente en hablar de «libertad» y «reciprocidad» cuando éstas beneficiaban al comercio británico. En caso contráiio, las nuevas ideas se olvidaban en beneficio de una postura más tradicional: si una balanza comercial favorable significaba claramente poder, había que «ayudar» al fiel de dicha balanza a inclinarse hacia el lado británico y no esperar, confiando en que la libertad estableciese un equilibrio justo.

\section{CONCLUSIONES}

En estas condiciones y teniendo en cuenta la fortaleza británica y la rapidez con que consiguió salir de su aislamiento internacional - firma de la Triple Alianza-, las posibilidades españolas de llegar a un acuerdo satisfactorio y aprovechar la derrota británica en la guerra de las colonias

${ }^{18}$ PRO: FO 72/5. Observations on the British trade to Spain. 1785. 
americanas eran mínimas. El espejismo inicial de una Gran Bretaña debilitada pronto se desvaneció, pero los deseos ilustrados de animar su economía, siguiendo los patrones propuestos entre otros por Ward, intentaron imponerse por encima de todo. Floriblanca se mantuvo inflexible en su solicitud de una mayor «reciprocidad» a cambio de concesiones a los británicos, provocando que durante años las negociaciones se convirtieran en un círculo vicioso, en el que se intercambiaban «propuestas» y "contrapropuestas" sin ningun avance significativo. La rigidez en las posturas de los negociadores, junto a otras dificultades externas, como el conflicto en la costa de los Mosquitos, la crisis holandesa entre Francia y Gran Bretaña, las frecuentes enfermedades de Floridablanca o la locura de Jorge III, propiciaron que Liston tuviese que abandonar la península hacia su nuevo destino sin haber conseguido la firma del tratado. Poco tiempo después moría Carlos III. La llegada al trono de un nuevo Monarca sembraría dudas en el nuevo embajador británico, Eden, sobre las posibilidades de Floriblanca para mantenerse en el poder y las consecuencias que para los intereses británicos podía tener una nueva orientación de la política exterior española. Sin embargo, Carlos IV mantuvo a Floridablanca en el puesto de primer secretario, siendo un acontecimiento exterior, el estallido de la Revolución francesa, el anuncio del inicio de un nuevo período en las relaciones entre las naciones europeas.

Poco antes de que comenzasen las guerras contra la Francia revolucionaria, el estallido de la crisis de Nutka (1790) en el Pacífico Norte proporcionaba una última prueba de la recuperación británica y de su posición de primera potencia, frente a una España cuyas glorias imperiales eran ya historia lejana. Se asistió a una reedición de la vieja polémica sobre los derechos al libre ejercicio de la navegación, el comercio y la pesca, defendidos por los británicos, y las pretensiones españolas de control sobre unos territorios que no eran capaces de ocupar y defender. Los españoles no pudieron controlar el conflicto desatado cuando intentaron evitar la penetración británica en el comercio de pieles y la pesca en una zona que, aunque alejada, consideraban parte del Virreinato de Nueva España. Gran Bretaña jugó muy fuerte, mostrándose dispuesta a ir a la guerra, mientras España, una vez más aunque ahora por otras causas, tenía problemas para lograr el apoyo de su aliada Francia. El Convenio que cerró la crisis concedía a los británicos importantes indemnizaciones y abría a sus barcos la costa americana, desde el norte de California hasta Alaska. Gran Bretaña empezaba a recoger los frutos de su labor diplomática durante los últimos años, consiguiendo pasar del 
aislamiento a encabezar la Triple Alianza. En cuanto a España, los cambios acontecidos en Francia hacían cada vez más inviable una alianza, los Pactos de Familia, que había sido el pilar central de la política exterior española durante casi un siglo. 